\title{
Embolic myocardial infarction due to coronary artery aneurysm in a patient with Loeys-Dietz syndrome
}

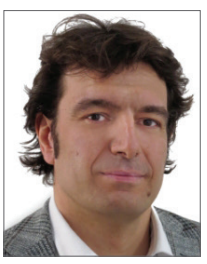

Alberto Cereda ${ }^{1 *}$, MD; Andrea Garascia ${ }^{1}$, MD; Paola Sormani $^{1}$, MD; Silvio Klugmann ${ }^{1}$, MD; Diana Artioli², MD; Francesco Soriano ${ }^{1}$, MD; Jacopo Andrea Oreglia ${ }^{3}$, MD

1. Cardiovascular Department, Niguarda Hospital, Milan, Italy; 2. Radiology Department, Niguarda Hospital, Milan, Italy; 3. Interventional Cardiology Unit, Luigi Sacco Hospital, Milan, Italy

This paper also includes supplementary data published online at: http://www.pcronline.comx/eurointervention/98th_issue/11

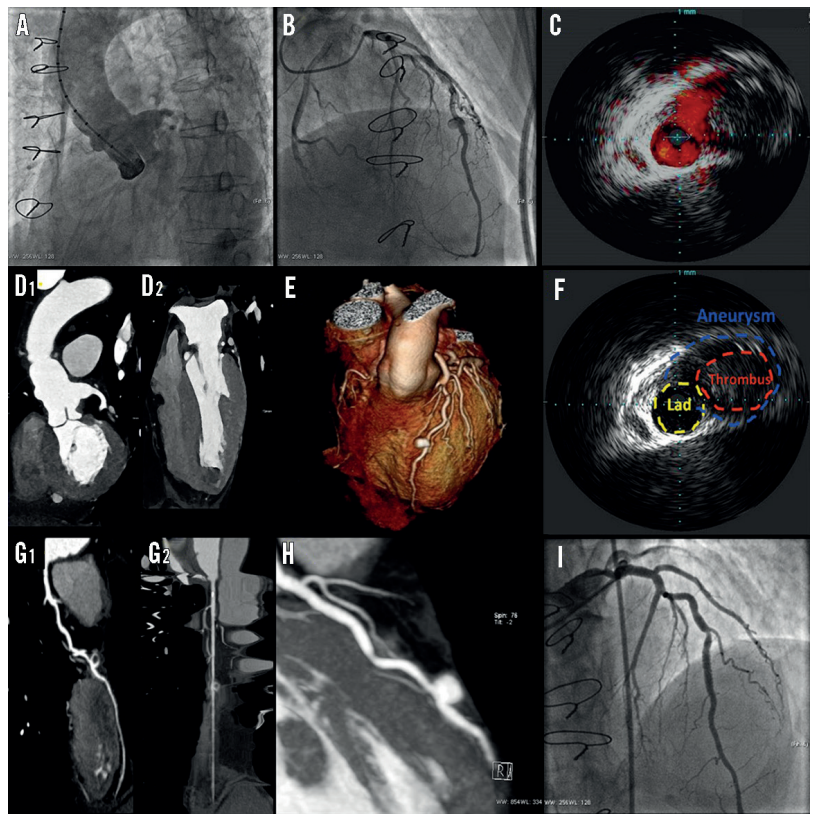

A 54-year-old male patient was admitted for non-ST-elevation acute coronary syndrome (NSTE-ACS). His medical history revealed previous normal coronary angiography, David's valve-sparing surgery performed one year ago, and a diagnosis of a TGFBR2-associated Loeys-Dietz syndrome. Emergency catheterisation showed evidence of a normal ascending aorta with an ostial aneurysm of $15 \mathrm{~mm}$ of the re-implanted LCA, a saccular pouching of the middle LAD and a non-occlusive luminal thrombus of the apical segment of the LAD with a TIMI grade 3 flow (Panel A, Panel B). IVUS showed diffused intimal thickness of the LAD with a vessel dilatation of $7 \times 10 \mathrm{~mm}$ and features highly suggestive of an aneurysm, partially obliterated by a round-shaped thrombus (Panel C, Panel F). A cardiac CT scan highlighted an apical ventricular thrombus, already suspected on echocardiogram, and the presence of a true aneurysm with an area of soft tissue density consistent with a thrombus of $6 \mathrm{~mm}$ (Panel D, Panel E, Panel G, Panel H). The coronary aneurysm was deemed suitable to be sealed with a $3.5 \times 16 \mathrm{~mm}$ PTFE-covered stent (Panel I), and IVUS confirmed the complete apposition of the covered stent and exclusion of the aneurysm with no residual filling. The postoperative course was uneventful and the patient was discharged on DAPT plus warfarin. Management of an ACS due to an embolic coronary aneurysm in the context of an overlapping syndromic frailty of vessels and atherosclerotic process is challenging. A multidisciplinary clinical and imaging approach is essential to provide successful treatment (Moving image 1-Moving image 10).

\section{Conflict of interest statement}

The authors have no conflicts of interest to declare.

\section{Supplementary data}

Moving image 1. Aortography.

Moving image 2. Angiography 1.

Moving image 3. Angiography 2.

Moving image 4. Angiography 3.

Moving image 5. Cardiac CT aneurysm.

Moving image 6. IVUS 1.

Moving image 7. IVUS 2 .

Moving image 8. Angiography post-PTFE stent 1.

Moving image 9. Angiography post-PTFE stent 2.

Moving image 10. Angiography post-PTFE stent 3.

*Corresponding author: ASST Grande Ospedale Metropolitano Niguarda, Piazza dell'Ospedale Maggiore, 3, 20162 Milan, Italy. E-mail: alberto.cereda@ospedaleniguarda.it 